\title{
A reason to stay healthy: The role of meaning in life in relation to physical activity and healthy eating among adolescents
}

Journal of Health Psychology 2015, Vol. 20(5) 473-482 (C) The Author(s) 2015

Reprints and permissions: sagepub.co.uk/journalsPermissions.nav DOI: $10.1177 / 1359105315576604$ hpq.sagepub.com

(S)SAGE

\author{
László Brassai', Bettina F Piko² and Michael F \\ Steger ${ }^{3,4}$
}

\begin{abstract}
The present longitudinal study investigated the incremental contribution of meaning in life to sustaining health-promoting behaviors, after controlling for well-being and health values among East-European adolescents $(N=456)$. Time I responses on presence of meaning, search for meaning, well-being, and health values were used to predict levels of healthy eating and physical activity 13 months later. All independent variables significantly predicted engagement in healthy eating and physical activity. Presence of meaning and search for meaning were the most robust predictors, and the interaction of them predicted additional variance in healthy eating among boys and physical activity among girls.
\end{abstract}

\section{Keywords}

adolescence, eating behavior, exercise behavior, life meaning, values, well-being

\section{Introduction}

Healthy eating and physical activity are key behaviors to sustain health and forestall the development of health-compromising conditions, such as obesity and heart disease (Biddle et al., 2004). Both activities require some degree of discipline, commitment, and constraint, suggesting that cognitive and motivational factors should play some role in their maintenance (Sutton, 2004). Meaning in life (MIL) also has been defined along cognitive and affective lines (Steger, 2009), making it a natural candidate for health research. Research reveals a strong interconnection between health and the existential dimension of personality (Frankl, 1963). In a developmental context, theoretical and empirical works demonstrate that the existential aspects of human life, especially the degree to which they judge their lives to be meaningful, may have a protective role not only for the health of both adults and the elderly but also for the health of adolescents (Erikson, 1968; Steger et al., 2012).

\footnotetext{
'Psychopedagogical Consulting Center, Romania

2University of Szeged, Hungary

${ }^{3}$ Colorado State University, USA

${ }^{4}$ North-West University, South Africa
}

\section{Corresponding author:}

László Brassai, Department of Psychology,

Psychopedagogical Consulting Center, Covasna, 520004

Saint George, Victor Babes I5/C, Romania.

Email: laszlobrassai@gmail.com 
Valuing health (health values (HV)) is an appealing, proximal predictor of health behaviors because it is specific to health. Certainly, adolescents may view health to be salient, but it would be useful to identify more general, distal predictors of health behaviors as well. General protective factors, such as well-being (WB) and MIL, may be leading indicators of attachment to health behaviors. WB may provide a sufficient emotional-motivational basis for commitment to and engagement in healthpromoting behaviors (Forgas, 2013), and the appreciation that one's life has meaning may lead people to appreciate and to invest in their lives, fostering their investment in health (Steger et al., 2014).

The relationships between MIL, WB and VH are best viewed as reciprocal. WB has been theorized to be a direct consequence of a life devoted to a purpose in life (Ryff and Singer, 1998). However, MIL theorists have contended that MIL might account for associations between different dimensions of WB (Zika and Chamberlain, 1992). Otherwise, values in general are thought to play an important role in the development of MIL, and specific domains that people value should be represented robustly in people's meaning system (Steger and Frazier, 2005). People who place high VH also should incorporate health into that which makes their lives feel meaningful.

There is less support for a positive correlation between MIL and physical health, although some support is emerging establishing positive links between MIL and neuroendocrine, immunological and cardiovascular markers of health (Bower et al., 1998), as well as perceived health (Steger et al., 2009a). One possible reason is a reduction of the harmful effects of stress (Park, 2013), as reflected in less sympathetic nervous system reactivity and better immune functioning (Bower et al., 2003).

However, research on adolescents shows that meaning is not only associated with better health (Brassai et al., 2011) but also with reduced exposure to health-risking behaviors (Newcomb and Harlow, 1986; Nicholson et al., 1994). Thus, engagement in health-promoting behaviors seems to be a strong candidate for understanding the link between MIL and health.

There are several different models and theories of what MIL is. According to these, MIL consists of a sense of order (Battista and Almond, 1973) or a sense of purpose (Emmons, 2003). Multidimensional definitions of MIL often combine these two dimensions with an affective dimension referencing people's fulfillment in their lives. For example, Reker and Wong (1988) defined meaning in terms of the ability to perceive order and coherence in one's existence, along with the pursuit and achievement of goals, and feelings of affective fulfillment arising from such coherence and pursuits. Thus, in this study, we focused on the overarching concept of meaning, which we understand to incorporate both comprehension and purpose (Steger, 2009).

Previous research on adolescents focused on the relationship between MIL and health risk behaviors suggested that MIL played a role in protecting adolescents from drug use (Nicholson et al., 1994) and heavy drinking (Newcomb and Harlow, 1986). Assessing relation of MIL with health-protective behavior as well, to our best knowledge, only two cross-sectional studies provided findings about positive associations thus far (Brassai et al., 2012; Steger et al., 2014).

Another oversight in the literature is that it has focused mainly on the relative presence or absence of meaning. This oversight underscores an important difference between the presence of meaning (POM) and the search for meaning (SFM) in life, which may be defined "as strength, intensity, and activity of people's desire and efforts to establish and/or augment their understanding of the meaning, significance, and purpose of their lives" (Steger et al., 2008a: 200). The SFM has long been viewed as a promotional motivational force directed to finding personal meaning and purpose for life, thus giving SFM an important role in development (Erikson, 1968; Frankl, 1963).

Indeed, in adult samples, SFM is often positively related to distress and negatively related to WB (Steger et al., 2008a). At the same time, 
earlier years appear to be marked by a youthful SFM (Steger et al., 2009b) and may be positively correlated with healthy eating and physical activity among adolescents (Brassai et al., 2012). It is likely that the young people's high level of SFM is more developmentally normal and positive, representing a high level of motivation to understand and integrate their lives in meaningful ways, which might possibly lead them to be more actively engaged in activities that make their lives meaningful.

One way to reconcile contrasting views of SFM is by examining who is searching for meaning (Steger et al., 2008a). Among adolescents and young adults, high levels of POM can moderate the deleterious effects of SFM across, for example, positive psychological functioning (Steger et al., 2008b) and subjective health (Steger et al., 2009a). Thus, SFM may be positive for youths and may be part of a complex, open-minded existential exploration among those who seek to augment existing meaning systems (Martos et al., 2010).

If the health-promoting role of MIL represents an emerging health agenda, the healthprotective role of $\mathrm{VH}$ and $\mathrm{WB}$ is well established. The perceived VH (Lau et al., 1986) was developed to explore how people's health-relevant cognitions help them define their health situation, elicit health goals, and guide action (Verplanken and Holland, 2002). Initial research data show that $\mathrm{VH}$ by itself predicts the performance of preventive health behaviors (Kristiansen, 1985), including physical activity and preference for healthier foods among adolescents (Costa et al., 1989). However, a major review concluded that WB has both longitudinal and causal relationships with health and health-relevant behaviors (Lyubomirsky et al., 2005). Emotional indicators of WB may increase one's personal resources, better equipping people to work toward health outcomes (Bagozzi and Edwards, 2000).

The purpose of this study was to evaluate the incremental contribution of MIL-over and above $\mathrm{VH}$ and $\mathrm{WB}$ - to predict engagement in health-promoting behaviors over the course of
1 year. Research on adolescents aiming to elucidate the relationship between MIL and health is still in its early stages and has focused predominantly on the reduction or cessation of addictive behavior (Brassai et al., 2011; Newcomb and Harlow, 1986; Nicholson et al., 1994), and overwhelmingly on POM, not on both POM and SFM. This research rarely has tested meaning's relation with health behaviors in the context of other related variables, and to the best of our knowledge, this is the first study of longitudinal links between MIL and health-promoting behaviors.

In addition, we hypothesized that adolescents who reported greater MIL, WB, and VH at baseline would report more physical activity and healthier eating at 12-month follow-up. Specifically, we hypothesized that (a) POM and SFM would each predict greater engagement in health-promoting behaviors 1 year later above and beyond $\mathrm{VH}$ and $\mathrm{WB}$ as independent factors and (b) POM and SFM would interact to predict engagement in health-promoting behaviors such that those scoring high on both scales would report the greatest engagement in healthpromoting behaviors. Finally, we also explored gender differences.

\section{Methods}

\section{Participants and procedure}

Data collection at Time 1 (T1) began in 2010 among native Hungarian-speaking students enrolled in secondary schools in Transylvania, Romania. Multistage sampling (choosing towns $>$ high schools $>$ classes) was used to obtain a sample consisting of 500 students. The sampling was based on randomly selected classes from each randomly selected high school. Participation in the study was voluntary and contingent upon parental permission. After ethical consideration by the institutional review board (IRB), parents' approval forms were given with a brief explanation of the objectives of the study and were placed in sealed envelopes and collected from each of the participating schools. Of 500 students aged 16-19 years 
who were initially recruited, 468 valid questionnaires were returned $\left(M_{\mathrm{age}}=16.5\right.$ years, standard deviation $(\mathrm{SD})=1.3$ years, $46.8 \%$ males). Thirteen months after the initial assessment, the sample was contacted again (T2), and valid questionnaires were obtained from 456 adolescents $\left(M_{\mathrm{age}}=17.6\right.$ years, $\mathrm{SD}=1.4$ years, $46.3 \%$ males), yielding a 97.4 percent retention rate. Drop-out rate was 2.6 percent $(N=12)$, with no significant age and gender influences. Trained mental health educators distributed the questionnaires to students prior to the start of class, along with a brief explanation and instructions. Survey completion time was approximately 40 minutes. Participants completed measures of healthy eating behavior and physical activity at both $\mathrm{T} 1$ and $\mathrm{T} 2$, and $\mathrm{VH}, \mathrm{WB}$, POM in life, and SFM in life at T1 only. For all the measures, published Hungarian versions were used.

\section{Measures}

Health-protective behaviors. We used the healthy eating behavior and physical activity subscales of the Survey of Personal and Social Development (Jessor et al., 2003). Participants rated their engagement in health-promoting behaviors on a 7-point scale from 1 (absolutely untrue) to 7 (absolutely true). Both the healthy eating behavior subscale (e.g. "Eating healthy snacks like fruit instead of candy") and the physical activity subscale (e.g. "Practicing different physical activities") were assessed using 3 items. Scores appeared to have acceptable internal consistency for the physical activity subscale (T1 $\alpha=.75$, T2 $\alpha=.77$ ), although scores on the healthy eating subscale had only marginally acceptable internal consistency (T1 $\alpha=.71, \mathrm{~T} 2 \alpha=.72$ ). Paired-samples $t$-tests indicated non-significant differences between $\mathrm{T} 1$ and $\mathrm{T} 2$ for healthy eating (all $t \mathrm{~s}<1.25$, all $p \mathrm{~s}>.15$ ) and physical activity scores (all $t \mathrm{~s}<2.73$, all $p \mathrm{~s}>.96$ ).

Valuing health. To measure HV, we used the Value on Health Scale (VHS; Costa et al., 1989), which was designed to assess the value or importance placed upon several aspects of health: fitness or being in good physical condition, a sense of energy or vigor, endurance or stamina, maintaining an appropriate weight, and resistance to illness. VHS items begin with a common stem (i.e. "How important is it to you ...") and are rated from 1 (not important) to 4 (very important). The VHS has satisfactory internal consistency $(\alpha=.77)$ and good convergent and discriminant validity as well as in Hungarian sample (Piko, 2005). The reliability of scores on the VHS in the current sample was acceptable $(\alpha=.78)$.

WB. We used the World Health Organization Well-Being Index (WHO-5; Bech, 1998). The 5 items of the scale contain affirmations regarding positive mood (good spirits, relaxation), vitality (being active and waking up fresh and rested), and general interests (being interested in things). Items are rated from 1 (not agree) to 4 (strongly agree). High scores indicate greater positive WB. The Hungarian adaptation confirmed the homogeneity of this instrument and excellent internal consistency (Susánszky et al., 2006). The reliability of scores on the WHO-5 in the current sample was acceptable $(\alpha=.79)$.

POM in life and SFM in life. We used the Meaning in Life Questionnaire (MLQ; Steger et al., 2006). The MLQ includes two 5-item subscales: POM and SFM. The POM subscale assesses the degree to which people feel that their lives are meaningful. The SFM subscale assesses individuals' motivation and desire to find or deepen the meaning in their lives. Items are rated on a 7-point scale ranging from 1 (absolutely untrue) to 7 (absolutely true), high scores indicate high POM and SFM. The two-factor structure of the MLQ has been replicated, and both subscales demonstrate a good internal consistency among a Hungarian representative sample (Martos and Konkolÿ and Thege, 2012). In the present sample, we obtained good internal consistency $(\alpha=.81$, POM; $\alpha=.83$, SFM).

Data analysis. Data were analyzed using SPSS 17. Descriptive statistics and gender differences 
Table I. Gender differences in healthy eating and physical activity and other protective factors.

\begin{tabular}{llllll}
\hline & $\alpha$ & Boys, $M(S D)$ & Girls, M (SD) & t-value & Effect size $^{\text {a }}$ \\
\hline Time I & & & & & \\
$\quad$ Healthy eating & .72 & $17.8(4.2)$ & $18.9(4.0)$ & $2.87^{* * * *}$ & $d=.27$ \\
Physical activity & .77 & $14.4(4.9)$ & $11.4(5.2)$ & $6.37^{* * *}$ & $d=.59$ \\
$\quad$ Health values & .68 & $16.1(2.2)$ & $17.3(2.3)$ & $6.02^{* * *}$ & $d=.56$ \\
Well-being & .69 & $19.1(3.3)$ & $16.5(2.9)$ & $8.58^{* * *}$ & $d=.80$ \\
Presence of meaning & .84 & $23.9(5.9)$ & $22(5.4)$ & $1.89 *$ & $d=.18$ \\
$\quad$ Search for meaning & .77 & $24.5(4.2)$ & $25.3(4.7)$ & $2.12^{*}$ & $d=.20$ \\
Time 2 & & & & & \\
$\quad$ Healthy eating & .71 & $17.3(4.0)$ & $19.3(4.3)$ & $4.34^{* * *}$ & $d=.41$ \\
Physical activity & .75 & $13.6(4.6)$ & $10.6(4.8)$ & $6.7 I^{* * *}$ & $d=.63$ \\
\hline
\end{tabular}

SD: standard deviation.

aEffect sizes are Cohen's $d: .20=$ small effect; $.50=$ medium effect; $.80=$ large effect. $* p<.05 ; * * p<.01 ; * * p<.00 \mathrm{I}$.

were assessed by applying Student's $t$-tests, using Cohen's $d$ to indicate effect size. Interrelationships between health behaviors and healthprotective factors were assessed by multiple regression analysis for boys and girls separately. We summarized only one analysis of the two regression models (boys and girls separately) instead of focusing on the mediator role of gender. After analyzing the linearity of the relationship between dependent and independent variables (plots of the observed vs predicted values) and the normality of distribution (with Shapiro-Wilk statistics), we applied a logarithmic transformation to decrease the deviation. There was no significant multicollinearity (variance inflation factor from 1.199 to 1.964 ), nor significant outliers (standard residuals from -1.89 to 2.73). Levels of autocorrelation were sufficient (Durbin-Watson statistics from 1.8 to 2.1).

\section{Results}

In the whole sample, the POM had a high, positive correlation and the SFM had a smallto-medium positive correlation with all the studied variables, and the correlation between POM and SFM was also small and positive. Furthermore, there was a medium-to-high positive correlation between VH and WB and other dependent variables. Between physical activity and healthy eating scores assessed at
T1, there were high, positive correlations. The T1 physical activity had a high, positive correlation with healthy eating scores in $\mathrm{T} 2$, and the $\mathrm{T} 1$ healthy eating also had a high positive correlation with physical activity assessed in T2. Calculating test-retest correlations of physical activity at T1 and T2 and of healthy eating scale at T1 and T2 using Pearson's correlation coefficients, we evaluated the temporal stability as moderate.

However, boys reported higher WB and girls reported placing greater $\mathrm{VH}$ (see Table 1). In addition, boys reported higher POM and higher levels of physical activity, whereas girls reported higher SFM and higher levels of healthy eating behavior. Effect sizes ranged from small (for MIL), medium (for $\mathrm{VH}$ and health-promoting behaviors), to large (for WB).

To test the incremental validity of MIL in predicting health-promoting behaviors, we used hierarchical multiple regression, controlling for $\mathrm{VH}$ and WB. Dates of independent variables measured in T1 were introduced and two parallel hierarchical multiple regressions were performed, with one using T2 healthy eating as the dependent variable, and the other using T2 physical activity (see Table 2). VH was included in step 1, WB was added in step 2, POM and SFM were added in step 3, and finally, the interaction between centered scores for POM and SFM were added in step 4. 
Table 2. Hierarchical multiple regression analysis predicting healthy eating and physical activity.

\begin{tabular}{|c|c|c|c|c|}
\hline \multicolumn{2}{|l|}{ Boys $(N=2 I I)$} & \multicolumn{3}{|c|}{ Girls $(N=245)$} \\
\hline Healthy eating & Physical activity & & & Physical activity \\
\hline SE & SE & $\beta$ & SE & SE \\
\hline
\end{tabular}

Step I

Health values

$\begin{array}{lrl} & .21 * * * & .11 \\ \Delta R^{2} & .07^{* *} & \\ F & 27.828\end{array}$

$\begin{array}{cc}.23 * * * & .12 \\ .09 * * & \\ 29.375 & \end{array}$

$\begin{array}{ccr}.33 * * * & .14 & .28 * * * \\ .12 * * * & & .09 * * * \\ 35.101 & & 31.509\end{array}$

.09

Step 2

Health values

Well-being

$\begin{array}{lrr} & .17^{* * * *} & .11 \\ & .27^{* * * *} & .09 \\ \Delta R^{2} & .12^{* * *} & \\ F & 35.115\end{array}$

$\begin{array}{ll}.25 * * * & .12 \\ .33 * * * & .13 \\ .13 * * * & \\ 36.338 & \end{array}$

$\begin{array}{rrrr}.29 * * * & .16 & .25 * * * & .06 \\ .26 * * * & .12 & .36 * * * & .07 \\ .10 * * * & & .11 * * * & \\ 32.945 & 34.199 & \end{array}$

Step 3

Health values

Well-being

Presence of meaning

Search for meaning

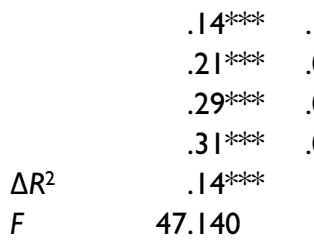

.13
.09
.07
.09

$\begin{array}{cc}.13^{* * * *} & .13 \\ .27^{* * *} & .12 \\ .30^{* * * *} & .09 \\ .26^{* * * *} & .07 \\ .12^{* * *} & \\ 34.998 & \end{array}$

$\begin{array}{cccc}.26 * * * & .18 & .26 * * * & .06 \\ .24 * * * & .11 & .31 * * * & .06 \\ .32 * * * & .09 & .34 * * * & .04 \\ .33 * * * & .10 & .36 * * * & .05 \\ .12 * * & & .13 * * * & \\ 33.132 & & 36.343 & \end{array}$

Step 4

Presence $\times$ Search

\begin{tabular}{|c|c|c|c|c|c|c|}
\hline & $.11 *$ & 更 & .08 & .24 & $.10^{*}$ & .11 \\
\hline$\Delta R^{2}$ & $.05^{*}$ & & .0 & .0 & $.04^{*}$ & \\
\hline$F$ & 13.545 & & 4.196 & 2.908 & 11.512 & \\
\hline Constant & $-8 .|2| * * *$ & & $-10.045 * * *$ & $-10.909 * * *$ & $-9.215^{* * *}$ & \\
\hline$R^{2}$ & $.38^{* * * *}$ & & $.34 * * *$ & $.34 * * *$ & $.37 * * *$ & \\
\hline
\end{tabular}

$\beta$ : standardized beta coefficient; SE: standard error.

Changes in the $R^{2}$ are based on the hierarchical F-test.

$*_{p}<.05 ; * * p<.01$; ***p $<.001$.

All four health indicators showed significant protective effects in relation to health-promoting behaviors among both boys and girls. The two MIL variables explained additional significant variance in healthy eating and physical activity. In addition, there appeared to be a modest trend toward stronger relationships between MIL variables and health-protective behaviors for girls (standardized beta-coefficients $(\beta s=.24-.36)$ than for boys $(\beta s=.13-.33)$.

Finally, simple main effects of MIL on healthy eating and physical activity scores were explored, focusing on possible differences between +1 SD and -1 SD of SFM with POM at +1 SD. In decomposing this interaction, it appeared that SFM amplified the positive relation between POM and healthy eating for boys $(\beta=.11, p<.05)$ and between POM and physical activity for girls $(\beta=.10, p<.04)$. Thus, there appeared to be a multiplicative interaction between POM and SFM in these instances.

\section{Discussion}

Regarding young people as resources to be developed and not as problems to be managed has become a more dominant view in the last decades (Roth et al., 1998), calling attention to the importance of promoting positive developmental trajectories rather than merely preventing negative developmental outcomes (Mulye 
et al., 2009). This perspective is inherently compatible with existential-humanistic models of health. Existential-humanistic models are highly focused on such accounts. Among the variables found in such models, MIL holds potential as a general, distal predictor of positive approaches to health (Steger et al., 2014).

In this study, we focused on testing the incremental validity of MIL for predicting engagement in health behaviors above and beyond the established constructs of $\mathrm{VH}$ and WB. The results indicated that all these variables predicted engagement in healthy eating and physical activity 1 year later, with the meaning variables having particularly robust predictive power. These findings provide several new and potentially important insights into the emergence of health in adolescence.

This study provided three important advances over previous research. First, unlike previous research, there was more than a yearlong gap between the assessment of MIL and health-promoting behaviors. Thus, we were able to extend previous research by showing the long-term health benefits of MIL, over and above other established predictors (Bagozzi and Edwards, 2000; Costa et al., 1989; Lau et al., 1986; Lyubomirsky et al., 2005; Ritt-Olson et al., 2004).

Second, previous research has focused mainly on the POM, with very little attention paid to SFM (Brassai et al., 2011; Newcomb and Harlow, 1986; Nicholson et al., 1994). The present longitudinal study is the first to show that SFM can serve as a positive factor in health-promoting activities among adolescents. Although previous research has found that SFM is sometimes positively related to $\mathrm{WB}$ in some cultures (Steger et al., 2008b) and age groups (Steger et al., 2009b), this study showed that it is positively related to healthy eating and physical activity as well. Thus, when SFM is grounded in people's aspirations and insights to overcoming life's challenges_-particularly in the formative years of adolescence - the feeling that one's life could have gone better may serve as a source of growth-oriented motivation to work toward improvement. Based on this, more attention should be paid to helping adolescents to engage in a productive SFM in life.

Third, we examined the interaction of POM and SFM in relation to adolescent health behaviors. Positive SFM was anticipated to occur among those who had already found meaning, whereas negative SFM was anticipated to occur among those who were without MIL (Steger et al., 2009a). Research has generally borne this hypothesis out (Steger et al., 2008a, 2008b). Only one other study has looked at this interaction in the context of health, among adult smoking cessation patients (Steger et al., 2009b). This study is the first to examine this interaction among adolescents. This examination revealed potentially important gender effects indicating that SFM strengthened the beneficial effects of POM in predicting healthy eating among boys, and physical activity among girls.

The role of gender in the link between MIL variables and health behaviors is uncertain, but it plays a well-established role in health behaviors. Therefore, we preferred a comparative analysis based on separate male and female regression models in order to pinpoint possible differences in their predictor structures, instead of testing the mediating role of gender. In concordance with other research on WB and HV, boys reported greater $\mathrm{WB}$ and girls reported greater VH (Räty et al., 2005; Ritt-Olson et al., 2004). Gender differences in MIL were substantially smaller than other gender differences in the present study. In contrast to previous results (Steger et al., 2009b), in our sample, boys reported higher levels of POM and girls reported higher levels of SFM. Predictor effects on health-promoting behaviors also differ by gender (Bagozzi and Edwards, 2000; Piko, 2005; Piko and Brassai, 2009; Vegar et al., 2011). In our sample, the strongest predictors for boys were the MIL variables, whereas all variables appeared similarly predictive for girls.

Although this research provided important empirical evidence of the health-protective role of $\mathrm{WB}, \mathrm{VH}$, and MIL, future research is necessary to address limitations in this study. One limitation was the reliance on self-report questionnaires to indicate key variables. It would be 
desirable to use behavioral measures to verify actual engagement in healthy eating and physical activity. In addition, we did not assess physical health directly, but only behaviors that are consistently linked with health. Direct health assessment would improve this line of research. Because of practical constraints, we were unable to administer duplicate questionnaires at both time points. This means we could not test whether the psychological factors predicted health-promoting behaviors above and beyond baseline levels of these activities. Likewise, with only two data points and a limited number of variables, we were unable to test whether other variables mediated the relationship between psychological variables and healthpromoting behaviors. A larger and longer longitudinal study would also allow testing potential age and social differences in psychological factors and health-promoting behaviors.

\section{Funding}

This research was supported by the following grant to the first author: the Domus Hungarica Scientiarum et Atrium 2013/I provided by the Hungarian Academy of Sciences (Hungary). These funds had no role in the study design; collection, analysis, or interpretation of the data; the writing of the article; or the decision to submit the article for publication.

\section{References}

Bagozzi RP and Edwards EA (2000) Goal-striving and the implementation of goal intentions in the regulation of body weight. Psychology \& Health 15: 255-270.

Battista J and Almond R (1973) The development of meaning in life. Psychiatry: Journal for the Study of Interpersonal Processes 36: 409-427.

Bech P (1998) Quality of Life in the Psychiatric Patient. London: Mosby-Wolfe.

Biddle SJ, Gorely T and Stensel DJ (2004) Healthenhancing physical activity and sedentary behaviour in children and adolescents. Journal of Sport Science 22: 679-701.

Bower JE, Kemeny ME, Taylor SE, et al. (1998) Cognitive processing, discovery of meaning, CD4 decline, and AIDS-related mortality among bereaved HIV-seropositive men. Journal of Consulting and Clinical Psychology 66: 979-986.
Bower JE, Kemeny ME, Taylor SE, et al. (2003) Finding positive meaning and its association with natural killer cell cytotoxicity among participants in a bereavement-related disclosure intervention. Annals of Behavioral Medicine 25: 146-155.

Brassai L, Piko B and Steger MF (2011) Meaning in life: Is it a protective factor for adolescents' psychological health? International Journal of Behavior Medicine 18: 44-51.

Brassai L, Piko B and Steger MF (2012) Existential attitudes and Eastern European adolescents' problem and health behaviors: Highlighting the role of the search for meaning in life. The Psychological Record 62: 719-734.

Costa FM, Jessor R and Donovan JE (1989) Value on health and adolescent conventionality: A construct validation of a new measure in problem-behavior theory. Journal of Applied Social Psychology 19: 841-861.

Emmons RA (2003) Personal goals, life meaning, and virtue: Wellsprings of a positive life. In: Keyes CLM and Haidt J (eds) Flourishing: Positive Psychology and the Life Well-Lived. Washington, DC: American Psychological Association, pp. 105-128.

Erikson EH (1968) Identity: Youth and Crisis. New York: W. W. Norton \& Co.

Forgas JP (2013) Belief and affect: On the mental pre-cursors of health-related cognition and behaviour. Journal of Health Psychology 18: 3-9.

Frankl VE (1963) Man's Search for Meaning: An Introduction to Logotherapy. New York: Square Press; Pocket Books.

Jessor R, Costa FM and Turbin T (2003) Survey of Personal and Social Development. Boulder, CO: Colorado University Press, Institute of Behavioral Science.

Kristiansen CM (1985) Value correlates of preventive health behavior. Journal of Personality and Social Psychology 49: 748-758.

Lau RR, Hartmen KA and Ware JE Jr (1986) Health as value: Methodological and theoretical considerations. Health Psychology 5: 25-43.

Lyubomirsky S, King LA and Diener E (2005) The benefits of frequent positive affect. Does happiness lead to success? Psychological Bulletin 131: 803-855.

Martos T and Konkolÿ Thege B (2012) Those who search and those who find-Assessing the presence of and search for the meaning in life with the Hungarian version of the meaning in life 
questionnaire. Hungarian Review of Psychology 67: 125-149 (in Hungarian).

Martos T, Konkolÿ Thege B and Steger MF (2010) It's not only what you hold, it's how you hold it: Dimensions of religiosity and meaning in life. Personality and Individual Differences 49: 863-868.

Mulye TP, Park MJ, Nelson CD, et al. (2009) Trends in adolescent and young adult health in the United States. Journal of Adolescent Health 45: 8-24.

Newcomb MD and Harlow LL (1986) Life events and substance use among adolescents: Mediating effects of perceived loss of control and meaninglessness in life. Journal of Personality and Social Psychology 51: 564-577.

Nicholson T, Higgins W, Turner P, et al. (1994) The relation between meaning in life and the occurrence of drug abuse: A retrospective study. Psychology of Addictive Behaviors 8: 24-28.

Park CL (2013) Trauma and meaning making: Converging, conceptualizations and emerging evidences. In: Hicks JA and Routledge $\mathrm{C}$ (eds) The Experience of Meaning in Life: Classical Perspectives, Emerging Themes, and Controversies. New York: Springer Press, pp. 61-76.

Piko BF (2005) Adolescents' health-related behaviors in the light of their value orientations. Substance Use \& Misuse 40: 735-742.

Piko BF and Brassai L (2009) The role of individual and familial protective factors in adolescents' diet control. Journal of Health Psychology 14: 810-819.

Räty LKA, Larsson G, Söderfeldt BA, et al. (2005) Psychosocial aspects of health in adolescence: The influence of gender, and general selfconcept. Journal of Adolescent Health 36: 530. e21-530.e28.

Reker GT and Wong PTP (1988) Aging as an individual process: Toward a theory of personal meaning. In: Birren JE and Bengtson VL (eds) Emergent Theories of Aging. New York: Springer, pp. 214-246.

Ritt-Olson A, Milam J, Unger JB, et al. (2004) The protective influence of spirituality and "Healthas-a-Value" against monthly substance use among adolescents varying in risk. Journal of Adolescent Health 34: 192-199.

Roth J, Brooks-Gunn J, Murray L, et al. (1998) Promoting healthy adolescents: Synthesis of youth development program evaluations.
Journal of Research on Adolescence 8: 423459.

Ryff CD and Singer B (1998) The contours of positive human health. Psychological Inquiry 9: $1-28$.

Steger MF (2009) Meaning in life. In: Lopez SJ (ed.) Oxford Handbook of Positive Psychology. 2nd ed. Oxford: Oxford University Press, pp. 679687.

Steger MF and Frazier P (2005) Meaning in life: One link in the chain from religion to well-being. Journal of Counseling Psychology 52: 574-582.

Steger MF, Bundick M and Yeager D (2012) Understanding and promoting meaning in life during adolescence. In: Levesque RJR (ed.) Encyclopedia of Adolescence. New York: Springer, pp. 1666-1677.

Steger MF, Fitch-Martin A, Donnelly J, et al. (2014) Meaning in life and health: Proactive health orientation links meaning in life to health variables among American undergraduates. Journal of Happiness Studies. Epub ahead of print 1 May. DOI: 10.1007/s10902-014-9523-6.

Steger MF, Frazier P, Oishi S, et al. (2006) The meaning in life questionnaire: Assessing the presence of and search for meaning in life. Journal of Counseling Psychology 53: 80-93.

Steger MF, Kashdan TB, Sullivan BA, et al. (2008a) Understanding the search for meaning in life: Personality, cognitive style, and the dynamic between seeking and experiencing meaning. Journal of Personality and Social Psychology 28: 199-228.

Steger MF, Kawabata Y, Shimai S, et al. (2008b) The meaningful life in Japan and the United States: Levels and correlates of meaning in life. Journal of Research in Personality 42: 660-678.

Steger MF, Mann J, Michels P, et al. (2009a) Meaning in life, anxiety, depression, and general health among smoking cessation patients. Journal of Psychosomatic Research 67: 353-358.

Steger MF, Oishi S and Kashdan TB (2009b) Meaning in life across the life span. Levels and correlates of meaning in life from emerging adulthood to older adulthood. Journal of Positive Psychology 4: 43-52.

Susánszky É, Konkolÿ Thege B, Stauder A, et al. (2006) Validation of the short (5-item) version of the WHO Well-Being Scale based on a Hungarian representative health survey (Hungarostudy 2002). Journal of Mental Health and Psychosomatics 3: 247-255 (in Hungarian). 
Sutton S (2004) Determinants of health-related behaviours: Theoretical and methodological issues. In: Sutton S, Baum A and Johnston M (eds) The Sage Handbook of Health Psychology. London: SAGE, pp. 94-126.

Vegar R, Holmen TL, Bauman A, et al. (2011) Factors predicting changes in physical activity through adolescence: The Young-HUNT Study, Norway. Journal of Adolescent Health 48: 616-624.
Verplanken B and Holland W (2002) Motivated decision making: Effects of activation and selfcentrality of values on choices and behavior. Journal of Personality and Social Psychology 82: 434-447.

Zika S and Chamberlain K (1992) On the relation between meaning in life and psychological well-being. British Journal of Psychology 83: 133-145. 